\title{
Multiscale analysis of instabilities in heterogeneous materials using ANM and multilevel FEM
}

\author{
Saeid Nezamabadi ${ }^{a}$, Hamid Zahrouni ${ }^{\mathrm{b}}$, Julien Yvonnet $^{\mathrm{c}}$ and Michel Potier-Ferry ${ }^{\mathrm{b}}$ \\ ${ }^{a}$ Laboratoire de Mécanique et Génie Civil (LMGC), Université Montpellier 2, UMR CNRS 5508, CC048 \\ Place Eugène Bataillon, 34095 Montpellier Cedex 05, France; ${ }^{b}$ Laboratoire d'Étude des \\ Microstructures et de Mécanique des Matériaux (LEM3), Université de Lorraine, UMR CNRS 7239, Ile \\ du Saulcy F-57045, Metz Cedex 01, France; ${ }^{c}$ Laboratoire Modélisation et Simulation Multi Échelle \\ (MSME), Université Paris Est, UMR CNRS 8208, 5 Bd Descartes, 77454 Marne-la-Vallée Cedex 02,
} France

\begin{abstract}
In this study, we propose a numerical technique which combines a perturbation approach (asymptotic numerical method) and a multilevel finite element analysis. This procedure allows dealing with instability phenomena in the context of heterogeneous materials where buckling may occur at both macroscopic and/or microscopic scales. Different constitutive relations are applied and geometrical non-linearity is taken into account at both scales. Numerical examples involving instabilities at both micro and macro levels are presented.

Dans cette étude, nous proposons une technique numérique qui combine une technique de perturbation (Méthode Asymptotique Numérique) et une analyse par éléments finis multi échelles. Cette procédure permet de traiter les problèmes d'instabilités dans le cadre des matériaux hétérogènes où ces instabilités peuvent survenir à la fois au niveau micro et au niveau macro. Différentes relations de comportement sont utilisées et la non linéarité géométrique est prise en compte à deux échelles. Des exemples numériques impliquant des instabilités au niveau micro et macro sont présentés.
\end{abstract}

Keywords: asymptotic numerical method; nonlinear homogenisation; multiscale finite element method; instabilities; buckling

Mots-clés: méthode asymptotique numérique; homogénéisation non linéaire; méthode d'éléments finis multi échelles; instabilités; flambage

\section{Introduction}

The instability phenomena in heterogeneous materials containing dispersions of multiple phases in the microstructure are of structural and/or material types. These phenomena can occur at both the macro and micro scales and may influence each other. Prior work in this domain has been carried out by Abeyaratne and Triantafyllidis (1984). They showed that unit elastomers with periodic holes lose rank-one ellipticity even though the elastomer possessed rank-one ellipticity. Afterwards, many authors have studied these phenomena in the heterogeneous materials; e.g. (deBotton, Hariton, \& Socolsky, 2006; Geymonat, Müller, \& Triantafyllidis, 1993;

*Corresponding author. Email: saeid.nezamabadi@univ-montp2.fr 
Lopez-Pamies \& Ponte Castañeda, 2005; Michel, Lopez-Pamies, Ponte Castanñeda, \& Triantafyllidis, 2007; Miehe, Schröder, \& Becker, 2002).

In our previous works (Nezamabadi, Yvonnet, Zahrouni, \& Potier-Ferry, 2009, 2010a), we have proposed a numerical method, called "the Multiscale-ANM technique", to solve non-linear problems in the framework of heterogeneous materials. This technique combines two numerical tools. The first one is the multiscale finite element method $\left(\mathrm{FE}^{2}\right)$ (Feyel, 2003) which is based on multiscale computational procedures. In this method, the macroscopic behaviour at every material point of the macrostructure is found by solving a non-linear finite element problem on the representative volume element (RVE) associated with the micro scale. The second tool concerns the numerical procedure to solve the resulting non-linear multiscale problem. It is based on the asymptotic numerical method (ANM) (Cochelin, 1994; Cochelin, Damil, \& Potier-Ferry, 2007). It consists of expanding the main variables of the problem into power series with respect to a path parameter. By comparison with Newton-Raphson procedure, ANM can be considered as a high-order predictor without the need of any iteration. Many applications of ANM to structural and fluid mechanics show the performance of this technique.

In the framework of the multiscale-ANM, since the microscopic and macroscopic non-linear problems to be solved are transformed into a sequence of linear problems, localisation and homogenisation tensors can be constructed for each linear local problem in the homogenisation context. We then compute the tangent stiffness matrix which is the same for all the linear local problems allowing a significant reduction of computation time since only one decomposition of this matrix is needed for the asymptotic steps. The length of each step is a posteriori estimated using the previously computed terms of the series. An algorithm which naturally adapts the step length can then be obtained. Hence, instability phenomena can be analysed with high accuracy and efficiency. In this paper, we revisit the multiscale-ANM technique, especially by applications to bending strength of beams. The capability of this method to deal with the local and global instabilities has been shown again through an application.

The layout of this paper is as follows: in Section 2, the formulation of micro-macro problem and the perturbation procedure applied to the multiscale problem are described briefly. In Section 3, accuracy and efficiency of the approach are evaluated through a numerical example involving instability phenomena.

\section{Multiscale formulation of the mechanical problem}

\subsection{Macroscopic and microscopic variational formulations}

Let $\Omega$ a domain in $\mathbb{R}^{\mathrm{d}}, \mathrm{d}$ being the space dimension, associated with a macroscopic structure and $\partial \Omega$ its external boundary, both in their reference configuration. The structure is subjected to prescribed displacements and forces on the disjoint complementary parts of the boundary $\partial \Omega_{u}$ (the Dirichlet boundaries) and $\partial \Omega_{t}$ (the Neumann boundaries), respectively. In the framework of a total Lagrangian formulation, the weak form of the macroscopic equilibrium equation can be written in the absence of body forces as follows:

Find $\overline{\mathbf{u}} \in \mathcal{S}(\Omega)$ satisfying the essential boundary conditions, i.e. $\overline{\mathbf{u}}=\tilde{\mathbf{u}}$ on $\partial \Omega_{u}$, with $\tilde{\mathbf{u}}$ being the prescribed displacements, and $\mathcal{S}(\Omega)$ the space of sufficiently smooth functions, such that:

$$
\int_{\Omega}^{t} \overline{\mathbf{P}}: \delta \overline{\mathbf{F}} d \boldsymbol{\Omega}=\lambda \int_{\partial \Omega_{t}} \overline{\mathbf{f}} \cdot \delta \overline{\mathbf{u}} d \Gamma \quad \text { in } \Omega,
$$

where $\overline{\mathbf{P}}$ is the first macroscopic Piola-Kirchhoff stress tensor, $\overline{\mathbf{F}}(\overline{\mathbf{F}}=\nabla \overline{\mathbf{u}}+\mathbf{I})$ denotes the macroscopic deformation gradient tensor and hence, $\delta \overline{\mathbf{F}}=\nabla \delta \overline{\mathbf{u}}$. $\overline{\mathbf{f}}$ represents the prescribed 
load on the external boundary $\partial \Omega_{t}, \lambda$ is a loading parameter and $\delta \overline{\mathbf{u}} \in \mathcal{S}^{0}(\Omega), \mathcal{S}^{0}(\Omega)$ being the space of all displacement fields vanishing on $\partial \Omega_{u}$ and sufficiently smooth on $\Omega$. Note that $\mathbf{A}: \mathbf{B}=A_{i j} B_{j i}$.

For the microscopic scale, the material is assumed to be heterogeneous with a periodic microstructure, characterised by a RVE that occupies a domain $\omega \in \mathbb{R}^{d}$ in its reference configuration. The weak form associated with the microscopic problem is:

Find $\mathbf{u} \in \mathcal{S}(\omega)$ satisfying the microscopic boundary conditions, i.e. Equation (6), such that:

$$
\int_{\omega}^{t} \mathbf{P}: \delta \mathbf{F} d \omega=0 \text { in } \omega,
$$

where $\delta \mathbf{u} \in \mathcal{S}^{0}(\omega)$, and $\mathcal{S}(\omega)$ and $S^{0}(\omega)$ being defined as previously and $\mathbf{u}$ is the microscopic displacement.

At the microscopic scale, we assume that the constitutive relations are known in each phase of the RVE. Generally, the material behaviour of each microstructural constituent (r) (e.g. matrix, inclusion, etc.) can be described by a relationship between the second Piola-Kirchhoff stress tensor, $\mathbf{S}$ and the Green-Lagrange strain tensor, $\gamma\left(\gamma=\frac{1}{2}\left({ }^{t} \mathbf{F F}+\mathbf{I}\right)\right)$ :

$$
\mathbf{S}=\mathcal{F}^{(r)}(\gamma)
$$

where $\mathbf{S}$ is related to $\mathbf{P}$ through $\mathbf{P}=\mathbf{F S}$. By this definition, we emphasise that the present framework is not specifically designed for any particular constitutive law; the microstructural material behaviour may be very complex and includes a physical and/or geometrical evolution of the microstructure, when modelled on the microstructural level. Moreover, in the context of the ANM, other constitutive laws (including inelastic behaviours) have been developed (see examples in hyperelasticity, viscoplasticity and plasticity in previous ANM papers; (Assidi, Zahrouni, Damil, \& Potier-Ferry, 2009; Descamps, Cao, \& Potier-Ferry, 1997; Nezamabadi, Zahrouni, \& Yvonnet, 2011; Zahrouni, Aggoune, Brunelot, \& Potier-Ferry, 2004)).

The macroscopic and microscopic problems are coupled through two main relations. First, as the constitutive relation is not explicitly given at the macroscopic level, the effective stress tensor $\overline{\mathbf{P}}$ is obtained by considering an average value of the microscopic stress field over the RVE. This relation is expressed as follows:

$$
\overline{\mathbf{P}}=\langle\mathbf{P}\rangle=\frac{1}{|\omega|} \int_{\omega} \mathbf{P} d \omega,
$$

where $|\omega|$ represents the volume of the considered RVE. The second relation concerns the mean value of the microscopic deformation gradient assumed in the form:

$$
\overline{\mathbf{F}}=\langle\mathbf{F}\rangle=\frac{1}{|\omega|} \int_{\omega} \mathbf{F} d \omega .
$$

This relation is deduced from the boundary conditions (BC) imposed on the RVE (Miehe, 2003). Generally, three main BC can be considered for the RVE as linear deformations, uniform tractions or periodic constraints. Here, we consider periodic conditions on the boundary of the RVE which can be recasted into the following form:

$$
\mathbf{u}^{+}-\overline{\mathbf{u}}=(\overline{\mathbf{F}}-\mathbf{I}) \cdot\left(\mathbf{X}^{+}-\overline{\mathbf{X}}\right) \text { on } \partial \omega
$$


where the exponents + and - are associated with node indices on opposite sides of the RVE. Note that the BC depend on the macroscopic deformation tensor $\overline{\mathbf{F}}$.

\subsection{Numerical algorithm}

The micro and macro formulations are described by the non-linear system of Equations (1)-(6). Solution of this non-linear multiscale problem is sought using the ANM. In the context of ANM, both variables of macroscale and microscale of the considered multiscale problem are expanded into power series. We propose to represent these variables in a mixed vector $\mathbf{U}=(\overline{\mathbf{u}}, \overline{\mathbf{P}}, \mathbf{u}, \mathbf{P}, \ldots)$. The perturbation technique is applied to $\mathbf{U}$ and to the load parameter $\lambda$, leading to the following expression:

$$
\left\{\begin{array}{l}
\mathbf{U}(a) \\
\lambda(a)
\end{array}\right\}=\left\{\begin{array}{c}
\mathbf{U}_{0} \\
\lambda_{0}
\end{array}\right\}+\sum_{p=1}^{N} a^{\mathrm{p}}\left\{\begin{array}{c}
\mathbf{U}_{\mathrm{p}} \\
\lambda_{\mathrm{p}}
\end{array}\right\}
$$

where $().(a)$ refers to quantities defined continuously with respect to a scalar path parameter $a$ to be defined, $(.)_{0}$ denotes a known initial solution such that $().(0)=(.)_{0}, N$ is the truncation order of the series, and $(.)_{\mathrm{p}}$ indicates a term at order " $\mathrm{p}$ " that has to be determined.

By using Equation (7), the problem (1) is transformed to a sequence of problems:

$$
\int_{\Omega}^{t} \overline{\mathbf{P}}_{\mathrm{p}}: \delta \overline{\mathbf{F}} d \mathbf{\Omega}=\lambda_{\mathrm{p}} \int_{\partial \Omega_{F}} \mathbf{f} \cdot \delta \overline{\mathbf{u}} d \Gamma \quad \forall p=1, \ldots, N .
$$

Since the macroscopic constitutive relation is unknown, it is needed to carry out a computation on the microscopic problem to extract it.

The microscopic problem is defined as follows:

$$
\begin{gathered}
\left\{\begin{array}{c}
\int_{\omega}{ }^{t} \mathbf{P}: \delta \mathbf{F} d \omega=0 \\
\mathbf{S}=\mathcal{F}^{(r)}(\gamma) \\
\mathbf{P}=\mathbf{F} \cdot \mathbf{S} \\
\left.\gamma=\frac{1}{2}{ }^{t} \mathbf{F} \cdot \mathbf{F}+\mathbf{I}\right) \\
\mathbf{F}=\nabla \mathbf{u}+\mathbf{I}
\end{array}\right. \\
+\mathrm{BC}: \mathbf{u}^{+}-\mathbf{u}^{-}=(\overline{\mathbf{F}}-\mathbf{I})\left(\mathbf{X}^{+}-\mathbf{X}^{-}\right) \text {on } \partial \omega .
\end{gathered}
$$

The ANM expansion of Equations (9) leads also to a sequence of linear problems which can be solved partly at each order by considering the coupling relations between macroscopic and microscopic problems. In this context, the obtained solution permits one to construct numerically a localisation tensor. Hence, one can obtain the following relation between microscopic and macroscopic deformation gradient at any order "p" (see Nezamabadi et al., 2009, 2010a):

$$
\mathbf{F}_{p}=\mathbb{A}, \mathbf{x}: \overline{\mathbf{F}}_{\mathrm{p}}+\mathbf{u}_{\mathrm{p}, \mathbf{X}}^{\mathrm{nl}}
$$

where $\mathbb{A}, \mathbf{x}$ is a fourth-order tensor identified as a localisation tensor and $\mathbf{u}_{\mathrm{p}, \mathbf{X}}^{\mathrm{n}}$ is a secondorder tensor. This latter is a residual term depending on the solutions of the computed orders before the order " $p$ ".

To obtain the homogenised constitutive relation, we consider the microscopic constitutive relation at order "p": 


$$
\mathbf{P}_{\mathrm{p}}=\mathfrak{U}^{(r)}: \mathbf{F}_{\mathrm{p}}+\mathbf{P}_{\mathrm{p}}^{\mathrm{nl}}
$$

where $\mathbb{G}^{(r)}$ represents the constitutive tensor associated with phase $(r)$ and $\mathbf{P}_{\mathrm{p}}^{\mathrm{nl}}$ is a residual term which depends on the solutions at the previous " $p-1$ " orders. By replacing the relation (10) in (11):

$$
\mathbf{P}_{\mathrm{p}}=\mathbb{U}^{(r)}: \mathbb{A}_{, \mathbf{X}}: \overline{\mathbf{F}}_{\mathrm{p}}+\mathbb{W}^{(r)}: \mathbf{u}_{\mathrm{p}, X}^{\mathrm{nl}}+\mathbf{P}_{\mathrm{p}}^{\mathrm{nl}}
$$

and by setting $\mathbf{P}_{\mathrm{p}}^{* \mathrm{nl}}=\mathbb{M}^{(r)}: \mathbf{u}_{\mathrm{p}, X}^{\mathrm{nl}}+\mathbf{P}_{\mathrm{p}}^{\mathrm{nl}}$ and $\mathbb{L}=\mathbb{T}^{(r)}: \mathbb{A}_{, \mathbf{X}}$, we obtain:

$$
\mathbf{P}_{\mathrm{p}}=\mathbb{L}: \overline{\mathbf{F}}_{\mathrm{p}}+\mathbf{P}_{\mathrm{p}}^{* \mathrm{nl}} .
$$

The effective stresses can be obtained at each order "p" by averaging the Equation (13):

$$
\overline{\mathbf{P}}_{\mathrm{p}}=\mathbb{L}: \overline{\mathbf{F}}_{\mathrm{p}}+\mathbf{P}_{p}^{\mathrm{nl}},
$$

where $\overline{\mathbb{L}}=\frac{1}{|\omega|} \int_{\omega} \llbracket d \omega$ and $\overline{\mathbf{P}}_{\mathrm{p}}^{\mathrm{nl}}=\frac{1}{|\omega|} \int_{\omega} \mathbf{P}_{\mathrm{p}}^{* \mathrm{nl}} d \omega$.

By introducing (14) in Equation (8), we obtain the final linear form of the macroscopic problem at order "p":

$$
\int_{\Omega}{ }^{t} \overline{\mathbf{F}}_{\mathrm{p}}: \overline{\mathbb{L}}: \delta \overline{\mathbf{F}} d \Omega=\lambda_{\mathrm{p}} \int_{\partial \Omega_{t}} \mathbf{f} \cdot \delta \overline{\mathbf{u}} d \Gamma-\int_{\Omega}{ }^{t} \overline{\mathbf{P}}_{\mathrm{p}}^{\mathrm{nl}}: \delta \overline{\mathbf{F}} d \Omega .
$$

The tangent modulus, $\overline{\mathbb{L}}$, is computed numerically from constructed problems at the microscopic level. The procedure is to solve the problem (15) which gives the displacements at the macrostructure $\overline{\mathbf{u}}$. Then, at each integration point, the tensor $\overline{\mathbf{F}}$ can be calculated which allows one to finish the computations of different variables at the microscopic level.

Details of the solving procedure of multiscale problems using ANM are given in our previous papers; (Nezamabadi et al., 2009, 2010a).

Furthermore, in the finite element procedure, the discretisation of the RVE (microscopic level) and of the macrostructure induces $p$ and $P$ integration points, respectively. The computational cost in terms of local and global variables is then of order $\mathcal{O}(p \times P)$. This cost increases quickly according to the structure size (number of elements) which is studied. Here, the parallelisation procedure is employed to reduce computational costs. For that purpose, computations associated with groups of integration points are distributed on several processors. As a result, computational costs are drastically reduced without developing specific algorithm.

\section{Numerical application to the plastic microbuckling of long fibre composites}

In our previous works cited above, the multiscale-ANM technique has shown its efficiency in dealing with the microscopic and/or macroscopic instabilities. In this work, we show once more the robustness of this method through a main example: the microbuckling of long fibre composites. As is well known (Grandidier, Casari, \& Jochum, 2012), the failure of these materials yields from a local fibre microbuckling and this instability is mainly governed by fibre waviness and matrix plastic behaviour. Nevertheless, the failure level is not a pure material property and it depends also on macroscopic structural quantities like specimen thickness or stacking sequence and it is not the same in bending and in pure compression(Wisnom, 
1991; Wisnom, Atkinson, \& Jones, 1997; Drapier, Grandidier, \& Potier-Ferry, 1999, 2001; Grandidier et al., 2012). In this paper, we only focus on plate thickness. To our knowledge, this thickness effect has not yet been described by a multiscale concurrent modelling.

The proposed problem has been discretised using two-dimensional finite elements in the plane stress framework. The macrostructure has been meshed with eight-node quadrangular elements whereas the microstructure has been meshed with nine-node quadrangular elements.

In the context of ANM, the truncation order $N$ of the series and the accuracy parameter $\delta$ which allows limiting the length of each asymptotic step in a continuation procedure, have to be determined. In all the tests presented here, we have solved the non-linear problems using ANM with $N=15$ and $\delta=10^{-8}$. For all the examples, Poisson's ratio is equal to .3.

The influence of microbuckling of the fibre with the initial imperfection on the macrostructure is assessed here by studying the bending of a beam made of a fibre-reinforced composite material. We consider a bidimensional representation of a laminate (see Figure 1), where $\mathbf{e}_{2}$ is the $90^{\circ}$ direction corresponding to the loading direction. The imperfection magnitude $\left(v_{0}\right)$ is constant through the thickness. One fibre is chosen as the microstructure and the periodic BC at microscopic scale as described in Equation (6) are used. For the applications that we target in the present work, we consider a linear Saint-Venant Kirchhoff constitutive relation for the fibre and an elastoplastic constitutive law based on the Ramberg-Osgood relation (see, e.g. Zahrouni, Potier-Ferry, Elasmar, \& Damil, 1998) for the matrix. The latter relation is written in the $3 \mathrm{D}$ case in the following form:

$$
E \gamma=(1+v) \mathbf{S}^{d}-(1-2 v) P \mathbf{I}+\frac{3}{2} \alpha\left[\frac{\mathbf{S}_{\mathrm{eq}}}{\sigma_{y}}\right]^{n-1} \mathbf{S}^{d}
$$

where $E, v, \alpha, n$ and $\sigma_{y}$ denote, respectively, the Young's modulus, Poisson's ratio, yield offset, hardening component and the yield stress. $P=-\frac{1}{3} \mathbf{S}: \mathbf{I}$ is the equivalent hydrostatic stress, $\mathbf{S}^{d}$ is the stress deviator defined by $\mathbf{S}^{d}=\mathbf{S}+P \mathbf{I}$. $\mathbf{S}_{\mathrm{eq}}$ is the von-Mises equivalent stress defined as follows:

$$
\mathbf{S}_{\mathrm{eq}}=\sqrt{\frac{3}{2} \mathbf{S}^{d}: \mathbf{S}^{d}}
$$

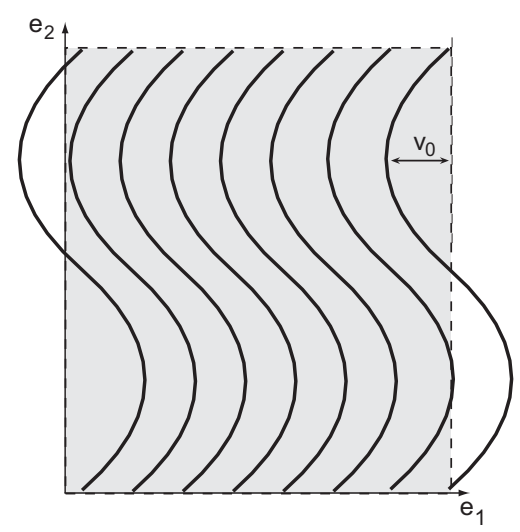

Figure 1. The microstructure of long fibre composite with the imperfection magnitude. 
Table 1. Characteristics of T300/914 material and data used for the plastic microbuckling study.

\begin{tabular}{lll}
\hline Fibre T300 (isotropic) & Matrix 914 (isotropic) & Imperfection \\
\hline$E_{f}=230 \mathrm{GPa}$ & $E_{m}=4500 \mathrm{MPa}$ & $\lambda_{0}=200 \pi \mu \mathrm{m}$ \\
$v_{f}=.3$ & $G_{m}=1600 \mathrm{MPa}$ & $v_{0}=3 \mu \mathrm{m}$ \\
$r_{f}=5 \mu \mathrm{m}$ & $v_{m}=.4$ & $\phi_{0}=3$ \\
$f=.6$ & $n=3$ & \\
& $\sigma_{y}^{m}=60 \mathrm{MPa}$ & \\
& $\varepsilon_{y}^{m}=2 \%$ & \\
\hline
\end{tabular}

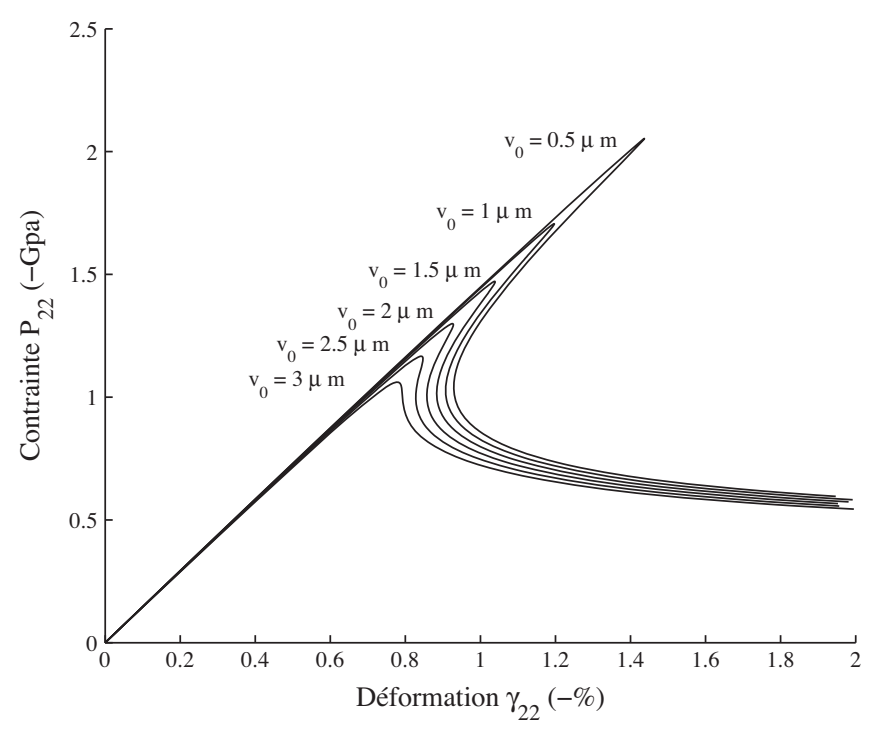

Figure 2. The macroscopic stress-strain diagrams for the different imperfection magnitudes.

The mechanical properties employed are shown in Table 1 which corresponds to a T300/ 914 composite. The parameters of the non-linear behaviour of the matrix are deduced from the work presented in Wisnom (1991). Figure 2 shows the responses under compression mode for different imperfection magnitudes (Drapier et al., 2001).

To show the influence of plastic microbuckling on the macrostructure response, we use a microstructure in which the imperfection magnitude of fibre is $3 \mu \mathrm{m}$. As the macrostructure, we consider a simply supported beam submitted to the force distribution $\lambda P$ on the top edge (see Figure 3); but, in order to evaluate the influence of the thickness on the failure of composites, two thicknesses of beam are studied: 1.5 and $3 \mathrm{~mm}$.

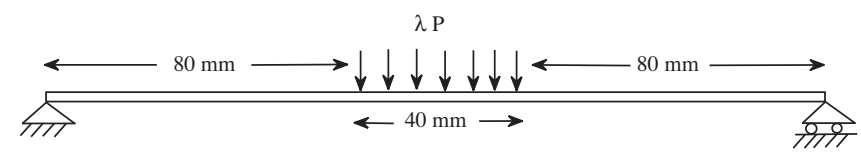

Figure 3. Geometries and $\mathrm{BC}$ of the rectangular beams made of the fibre reinforced composites; two considered beam thicknesses: 1.5 and $3 \mathrm{~mm}$, and $p=1 \mathrm{~N} / \mathrm{mm}$. 

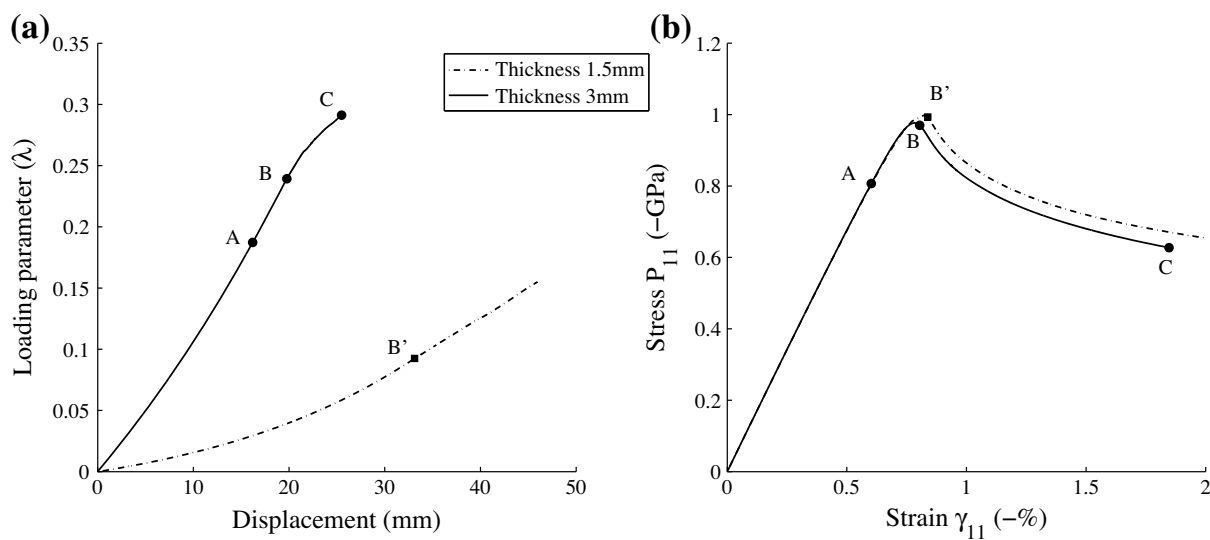

(c)

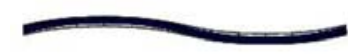

Point A

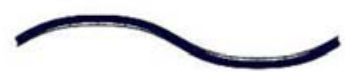

Point B

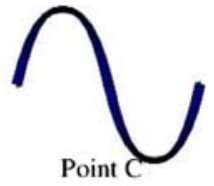

Figure 4. (a) The load-displacement diagrams of the plastic microbuckling problem for beam thicknesses of 3 and of $1.5 \mathrm{~mm}$; (b) The macroscopic stress-strain diagrams at the integration point located in the top middle (in a $3 \times 3$ integration point scheme) of the top middle element for beam thicknesses of $3 \mathrm{~mm}$ and of $1.5 \mathrm{~mm}$; and (c) Deformed shapes of microstructure at the considered integration point at points $\mathrm{A}, \mathrm{B}$ and $\mathrm{C}$ for beam thickness of $3 \mathrm{~mm}$, deformation scale $=10$.

The load-displacement responses for two beam thicknesses are presented in Figure 4(a), which shows the vertical displacement of the point located on the top middle of beam. This figure demonstrates the influence of microscopic instability on the macroscopic one: we can observe a slight change after the points B and B', the loss of ellipticity points. This is mainly caused by the occurrence of microscopic instabilities at this point. The deformed shapes of the microstructure at the integration point located in the top middle (in a $3 \times 3$ integration point scheme) of the top middle element at points A, B and C of the load-displacement curve for the beam with thickness of $3 \mathrm{~mm}$ (see Figure 4(a)) are presented in Figure 4(c). There is a significant difference between these deformed microstructural shapes despite the small variation of macroscopic load, which illustrates the relationship between local and global instabilities.

Figure 4(b) shows the macroscopic stress-strain diagrams at the considered integration point for the two beam thicknesses. In this figure, it can be observed that the thinner beam has the greater failure stress in comparison with the thicker beam (see points B and B' in Figure 4(b)). This phenomenon coincides with the fact shown in Drapier et al. $(1999,2001)$ that a decrease in thickness yields an increase in failure stress of fibre-reinforced composite. In the present case, the influence of beam thickness is moderate, but it can be larger for very thin plates (Grandidier et al., 2012). It is also worth noting that the points B and B', the maximum stress, correspond the points of the loss of ellipticity (see Figure 4(a) and (b)).

\section{Conclusion}

The multiscale-ANM technique that seems an efficient numerical technique in the context of heterogeneous materials to deal with instabilities which may occur on both the macro and micro levels, was revisited in this paper. This technique is the combination of the multiscale 
$\mathrm{FE}^{2}$ and the ANM. In this model, large displacements are taken into account in both macro and micro levels, and linear and non-linear constitutive relations can be implemented. The accuracy and the efficiency of the proposed procedure have also been shown in our previous papers; (Nezamabadi et al., 2009, 2010a).

The studied numerical example concerns the compression of a fibre-reinforced composite material with a microstructure in which we have considered only one fibre with initial imperfection. This example presents elastoplastic behaviour of the matrix and an elastic buckling of the fibre. The obtained results illustrate another time the capability of the proposed algorithm to deal with instabilities in the context of heterogeneous materials. Moreover, by considering two different values of the beam thickness at macroscopic scale, we show that the thinner the beam, the larger its strength for a beam under bending loads.

However, in this example, we have observed an accumulation of asymptotic steps and the loss of ellipticity close to the maximum load. These reasons reinforce the idea of extending our algorithms to a formulation based on the second-order homogenisation. Indeed, this formulation allows improving the conditioning of the problems that exhibit a loss of ellipticity and, also, dealing with the multiscale problems for which the microstructure size is of the same order as the characteristic structure size; (Nezamabadi, Zahrouni, Yvonnet, \& Potier-Ferry, $2010 \mathrm{~b}$ ). Ongoing work concerns applications of this technique to the modelling of local instabilities with loss of ellipticity.

\section{References}

Abeyaratne, R., \& Triantafyllidis, N. (1984). An investigation of localization in a porous elastic material using homogenization theory. Journal of Applied Mechanics, 51, 481-486.

Assidi, M., Zahrouni, H., Damil, N., \& Potier-Ferry, M. (2009). Regularization and perturbation technique to solve plasticity problems. International Journal of Material Forming, 2, 1-14.

Cochelin, B. (1994). A path following technique via an asymptotic numerical method. Computers and Structures, 53, 1181-1192.

Cochelin, B., Damil, N., \& Potier-Ferry, M. (2007). Méthode asymptotique numérique, (Asymptotic numerical method). Paris: Hermès Science.

deBotton, G., Hariton, I., \& Socolsky, E.A. (2006). Neo-Hookean fiber reinforced composites in finite elasticity. Journal of the Mechanic and Physics of Solids, 54, 533-559.

Descamps, J., Cao, H.L., \& Potier-Ferry, M. (1997). An asymptotic numerical method to solve large strain viscoplastic problems. In D.R.J. Owen, E. Oñate, \& E. Hinton (Eds.), Computational plasticity, fundamentals and applications (Vol. 13, pp. 393-400). Barcelona: C.I.M.N.E.

Drapier, S., Grandidier, J.C., \& Potier-Ferry, M. (1999). Towards a numerical model of the compressive strength for long fihre composites. European Journal of Mechanics A/Solids, 18, 69-92.

Drapier, S., Grandidier, J.C., \& Potier-Ferry, M. (2001). A structural approach of plastic micro buckling in long fibre composites: Comparison with theoretical and experimental results. International Journal of Solids and Structures, 38, 3877-3904.

Feyel, F. (2003). A multilevel finite element method $\left(\mathrm{FE}^{2}\right)$ to describe the response of highly nonlinear structures using generalized continua. Computer Methods in Applied Mechanics and Engineering, $192,3233-3244$.

Geymonat, G., Müller, S., \& Triantafyllidis, N. (1993). Homogenization of nonlinearly elastic materials: Microscopic bifurcation and macroscopic loss of rank-one convexity. Archive for Rational Mechanics and Analysis, 122, 231-290.

Grandidier, J.C., Casari, P., \& Jochum, C. (2012). A fibre direction compressive failure criterion for long fibre laminates at ply scale, including stacking sequence and laminate thickness effects. Composite Structures, 94, 3799-3806.

Lopez-Pamies, O., \& Ponte Castañeda, P. (2005). Second-order estimated for the macroscopic response and loss of ellipticity in porous rubbers at large deformations. Journal of Elasticity, 76, 247-287.

Michel, J.C., Lopez-Pamies, O., Ponte Castanñeda, P., \& Triantafyllidis, N. (2007). Microscopic and macroscopic instabilities in finitely strained porous elastomers. Journal of the Mechanics and Physics of Solids, 55, 900-938. 
Miehe, C. (2003). Computational micro-to-macro transitions for discretized micro-structures of heterogeneous materials at finite strains based on the minimization of averaged incremental energy. Computer Methods in Applied Mechanics and Engineering, 192, 559-591.

Miehe, C., Schröder, J., \& Becker, M. (2002). Computational homogenization analysis in finite elasticity: Material and structural instabilities on the micro- and macro-scales of periodic composites and their interactions. Computer Methods in Applied Mechanics and Engineering, 191(4), 4971-5005.

Nezamabadi, S., Yvonnet, J., Zahrouni, H., \& Potier-Ferry, M. (2009). A multilevel computational strategy for microscopic and macroscopic instabilities. Computer Methods in Applied Mechanics and Engineering, 198, 2099-2110.

Nezamabadi, S., Zahrouni, H., \& Yvonnet, J. (2011). Solving hyperelastic material problems by asymptotic numerical method. Computational Mechanics, 47, 77-92.

Nezamabadi, S., Zahrouni, H., Yvonnet, J., \& Potier-Ferry, M. (2010a). A multiscale finite element approach for buckling analysis of elastoplastic long fiber composites. International Journal for Multiscale Computational Engineering, 8, 287-301.

Nezamabadi, S., Zahrouni, H., Yvonnet, J., Potier-Ferry, M. (2010b). Multiscale second order homogenization using asymptotic numerical method, IV European Conference on Computational Mechanics, 16-21 May 2010, Paris, France.

Wisnom, M.R. (1991). The effect of specimen size on the bending strength of unidirectional carbon fibre-epoxy. Composite Structures, 18, 47-63.

Wisnom, M.R., Atkinson, J.W., \& Jones, M.I. (1997). Reduction in compressive strain to failure of unidirectional carbon fibre-epoxy with increasing specimen size in pin-ended buckling tests. Composites Science and Technology, 57, 1303-1308.

Zahrouni, H., Aggoune, W., Brunelot, J., \& Potier-Ferry, M. (2004). Asymptotic numerical method for strong nonlinearities. Revue Européenne des Eléments Finis, 118, 13-97.

Zahrouni, H., Potier-Ferry, M., Elasmar, H., \& Damil, N. (1998). Asymptotic numerical method for nonlinear constitutive laws. Revue Européenne des Eléments Finis, 7, 841-869. 